\title{
Assessment of Left Atrial Function in Patients with Non-ST-Segment Elevation Myocardial Infarction Using Two-Dimensional Speckle Tracking Echocardiography
}

\author{
Mansour M. Mostafa*, Wael M. Attia*, Mohamed O. Taha**, and *Mohamed N. Mahmoud \\ *Cardiology Department, Faculty of Medicine, Al-Azhar University and **National Heart Institute \\ (NHI), Cairo, Egypt \\ Corresponding author: Mohamed Nasem Mahmoud, E-mail: dr.mohamednassim@gmail.com
}

\begin{abstract}
Background: the left atrium (LA) plays a major role in Left ventricular (LV) performance. LA function is a surrogate marker of LV diastolic dysfunction. LA mechanical dysfunction occurs in LV systolic and diastolic dysfunction, coronary artery disease, myocardial infarction (MI), hypertension, aortic stenosis and cardiomyopathies. In the MI process, the contribution of the LA to LV function may increase if the cardiac myocytes are not affected by direct ischemia. However, myocyte necrosis or ischemia of the LA or significant LV systolic/diastolic dysfunction affects this booster effect. Assessment of LA size and function provides prognostic data for the outcome of patients with MI or ischemia. Aim of the Study: was to evaluate LA function in patients with non-ST-segment elevation myocardial infarction (NSTEMI) by two-dimensional speckle tracking echocardiography (2D STE). Patient and Methods: 60 patients with NSTEMI and 20 age-matched normal control individuals were enrolled in this study. Conventional echocardiographic parameters and global longitudinal strain rate (GLSR) were measured at left ventricular (LV) and LA segments. Results: Compared with healthy subjects, patients with NSTEMI had significantly increased LA volumes but significantly decreased LA emptying fraction and GLSR. LA-GLSR had significant correlations with the 2D Doppler echocardiographic parameters of LA function. In particular, global LA peak negative strain rate during early ventricular diastole (LA-GLSRe) was significantly correlated with both LA 2D Doppler echocardiographic parameters and LV contractile function. This could be suggested as a better indicator to evaluate LA function as a preferred parameter of STE. Conclusion: It could be concluded that two-dimensional speckle tracking echocardiography represented a non-invasive, relatively simple and reproducible technique to assess left atrial myocardial function in patients with NSTEMI.
\end{abstract}

Keywords: two-dimensional speckle tracking echocardiography, strain rate, non-ST-segment elevation myocardial infarction, left atrial function

\section{INTRODUCTION}

Myocardial infarction is a major cause of death and disability worldwide. MI may be the first manifestation of coronary artery disease (CAD) or it may occur, repeatedly, in patients with established disease ${ }^{(\mathbf{1})}$. Left atrial function is one of the most important clinical parameters of two-dimensional speckle tracking echocardiography (2D STE), which is an innovative tool for more comprehensive and reliable echocardiographic evaluation of myocardial function ${ }^{(2)}$.

LA function has been conventionally divided into three phases first, as a reservoir, the LA stores pulmonary venous return during $\mathrm{LV}$ contraction and isovolumetric relaxation. Secondly, as a conduit, the LA transfers blood passively into the LV. Thirdly, the LA actively contracts during the final phase of diastole and contributes between 15 and $30 \%$ of LV stroke volume. As a continuum of the LV, especially during diastole, its size and function are very much influenced by the compliance of the $\mathrm{LV}^{(3)}$.

Traditionally, assessment of LA function has been performed by measuring LA size or volume with two-dimensional (2D) echocardiography. Doppler echocardiographic measurements such as transmitral and pulmonary venous flow can also be used ${ }^{(4,5)}$. Currently, a method known as strain imaging is used for the quantitative assessment of myocardial deformation ${ }^{(6)}$. It has been shown that non-Doppler strain imaging is a feasible and reproducible method to assess LA function (7,8).

The LA function is closely related to the ventricular function throughout the cardiac cycle. During ventricular systole, the 
longitudinal shortening of the ventricular base increases the filling of the LA from the pulmonary veins. In diastole, the LA contributes to the filling of the LV through both active and passive emptying. Moreover, the LA emptying function might be strongly affected by LV diastolic properties due to the direct interaction of ventricular pressures through the open mitral valve during diastole ${ }^{(9)}$

Compared with Doppler and 2D echocardiography, 2D STE has the advantages of angle independence, and is also less affected by reverberations, side lobes or dropout artifacts. While this novel echocardiographic method has been frequently used to assess LV function ${ }^{(\mathbf{1 0})}$, it has been used to evaluate atrial function in normal subjects and in conditions with atrial dysfunction ${ }^{(11,12)}$.

The aim of the current work was to evaluate LA function in patients with non-ST-segment elevation myocardial infarction (NSTEMI) by twodimensional speckle tracking echocardiography (2D STE).

\section{PATIENTS AND METHODS}

This study included 60 patients with NSTEMI and 20 healthy individuals as control group attending at Al-Azhar University Hospitals. Approval of the ethical committee and a written informed consent from all the subjects were obtained. This study was conducted between Jan 2016 to September 2017.

Inclusion criteria: patients with non-STsegment elevation myocardial infarction (NSTEMI).

Exclusion criteria: Patients with atrial fibrillation or flutter, valvular heart disease (of mild or greater severity), and poor left atrial images were excluded.

Data regarding age, gender, body mass index (BMI) were obtained from all patients. All patients were subjected to:

A. Clinical evaluation including: History taking, General \& local examinations.

B. Laboratory investigations including: Lipids profile and plasma glucose level.

C. Conventional 2D and Doppler echocardiography studies were performed using the Philips IE33 equipped with a $3 \mathrm{~S}$ phased-array transducer (frequency range of 1.7-3.4 MHz). Echocardiographies of patients were performed within $48 \mathrm{hr}$. after NSTEMI. Cardiac dimensions were measured in accordance with recommendations of the American Society of Echocardiography. M-mode echocardiography was used to measure LV end-diastolic and end-systolic diameters. LV ejection fraction (LVEF) was calculated from apical four- and twochamber views, using the modified Simpson's rule. LA volumes were measured using the area-length method from apical four- and two-chamber views, according to the guidelines of the American Society of Echocardiography ${ }^{(\mathbf{1 3})}$. Left atrial maximum volume (LAVmax) was measured at the end of LV systole, just before the opening of the mitral valve, LA minimum volume (LAVmin) was measured at the end of LV diastole, right after the closure of the mitral valve, and LA reservoir volume (LAVres), (LAVres.): LAVmax. - LAVmin and LA ejection fraction (LAEF): (LAVmax)(LAmin.)/ $(\text { LAVmax })^{(\mathbf{1 4})}$. For 2D STE analysis, we obtained 2D gray-scale harmonic images in three apical planes (long axis of LV, four- and two-chamber). Three consecutive heart cycles were recorded and averaged. The frame rate was set between 60 and 90 frames per second $^{(\mathbf{1 5})}$. Echocardiograms were digitally stored and later analysed off-line using PHILPS Q Lab 8 A 16-segment LV model was obtained from the four- and twochamber, and long-axis recordings ${ }^{(16)}$. Two-dimensional strain software identified the endocardial border, and after tracing myocardial motion, was automatically tracked in each imaging view. Strain rate measurements from 16 segments were averaged to assess a LV global longitudinal parameter based on peak systole (LV-GLSRs), early diastole (LV-GLSRe), and late diastole (LVGLSRa). The LA myocardium was divided into six equidistant regions from apical four- and two-chamber views, while only three were analysed in the apical long-axis view because the remaining three in this view are part of the aortic valve and ascending aorta and not LA myocardium. The software generates 
strain rate curves for each atrial segment. Global strain and strain rate were also calculated by averaging values from 15 atrial segments.

\section{RESULTS}

This study included 60 patients with NSTEMI (mean age $54.42 \pm 9.24$ years, 38 males and 22

Table 1: Clinical features of patients with NSTEMI and controls

\begin{tabular}{|c|c|c|c|}
\hline Parameters & Cases $(n=60)$ & Controls $(n=20)$ & p-value \\
\hline Male, n (\%) & $38(63.3)$ & $14(70.0)$ & 0.588 \\
\hline Female, $\mathbf{n}(\%)$ & $22(36.7)$ & $6(30.0)$ & 0.588 \\
\hline Age (years) $($ Mean \pm SD) & $54.42 \pm 9.24$ & $36.35 \pm 9.95$ & $<0.001^{*}$ \\
\hline Weight (kg)Mean \pm SD. & $90.97 \pm 10.41$ & $87.65 \pm 8.95$ & 0.206 \\
\hline Height $(\mathrm{cm})$ Mean \pm SD. & $173.17 \pm 9.57$ & $174.60 \pm 5.93$ & 0.433 \\
\hline BMI $(\mathrm{kg} / \mathrm{m} 2)$ Mean \pm SD & $30.65 \pm 4.70$ & $28.56 \pm 2.43$ & $0.013^{*}$ \\
\hline Hypertension, n (\%) & $48(80.0)$ & $6(30.0)$ & $<0.001 *$ \\
\hline DM, n (\%) & $33(55)$ & $4(20.0)$ & $0.007^{*}$ \\
\hline Hyperlipidemia, n (\%) & $43(71.7)$ & $7(35.0)$ & $0.007 *$ \\
\hline Smoking, n (\%) & $31(51.7)$ & $11(55.0)$ & 0.796 \\
\hline
\end{tabular}

There were significant differences in clinical features, such as hypertension, diabetes and hyperlipidaemia between patients and healthy subjects Patients with NSTEMI had significantly increased LAVmax $(56.33 \pm 10.60 \mathrm{vs} 44.50 \pm 10.36 \mathrm{ml}, \mathrm{p}<0.001)$, LAVmin $(26.39 \pm 7.21 \mathrm{vs} 17.75 \pm$ $5.62 \mathrm{ml}, \mathrm{p}<0.001)$, and LAV res $(30.62 \pm 7.0 \mathrm{vs} 26.65 \pm 6.21 \mathrm{ml}, \mathrm{p}=0.001)$, but significantly lower in LAEF $(50.81 \pm 9.11$ vs $60.30 \pm 6.38 \%$, p <0.001), and LVEF $(56.22 \pm 6.29$ vs $60.40 \pm 3.99 \%, p=$ 0.001). 20 healthy subjects and 60 patients with NSTEMI were randomly selected for the assessment of LA-GLSRs, LA-GLSRe, LA-GLSRa, LV-GLSRs, LV-GLSRe and LV-GLSRa, respectively as seen in figures $(1,2)$



Figure 1: Measurement of global longitudinal left ventricular strain rate from an apical 4 camber view.

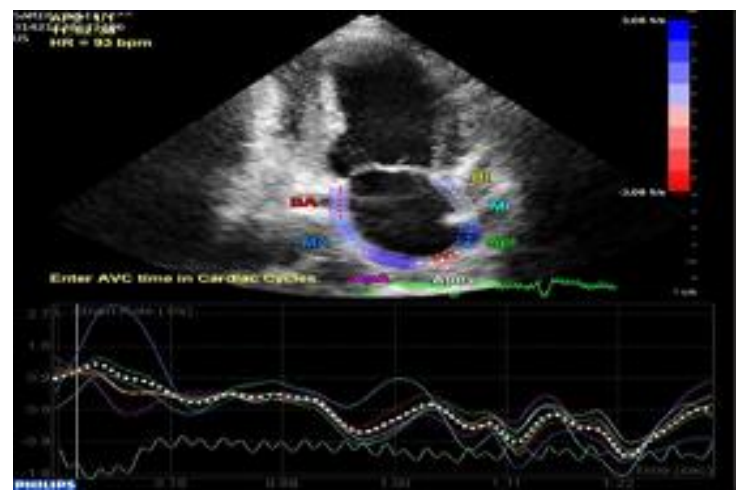

Figure 2: left atrial strain rate from an apical 2 chamber view.

The SR imaging echocardiographic variables of the normal and NSTEMI groups. Compared with the controls, patients with NSTEMI had significantly decreased LAGLS $(\mathrm{p}<0.001)$, LA-GLSRs ( $\mathrm{p}<0.001)$, LA-GLSRe ( $\mathrm{p}<0.001)$, LV-GLSRs $(\mathrm{p}<0.001)$, and LV-GLSRe $(\mathrm{p}=0.001)$, as seen in table 2 and figures $(3,4)$

Table 2: 2D STE parameters in patients with NSTEM and the controls

\begin{tabular}{|l|c|c|c|}
\hline & Cases $(\mathbf{n = 6 0})$ & Control $(\mathbf{n = 2 0})$ & $\mathbf{p}$ \\
\hline LAGLSs $(\%)$ & $22.53 \pm 5.20$ & $32.75 \pm 4.05$ & $<0.001^{*}$ \\
\hline LA-GLSRs & $1.36 \pm 0.45$ & $1.99 \pm 0.26$ & $<0.001^{*}$ \\
\hline LA-GLSRe & $-1.21 \pm 0.30$ & $-2.10 \pm 0.40$ & $<0.001^{*}$ \\
\hline LA-GLSRa & $-1.91-0.57$ & $-2.11 \pm 0.48$ & 0.168 \\
\hline LV-GLSRS & $-0.85 \pm 0.15$ & $-1.10 \pm 0.32$ & $<0.001^{*}$ \\
\hline LV-GLSRe & $1.05 \pm 0.54$ & $1.27 \pm 0.22$ & 0.001 \\
\hline
\end{tabular}






Figure 3: Comparison between the two studied groups according to LA-GLSRs, LA-GLSRe and LA-GLSRa

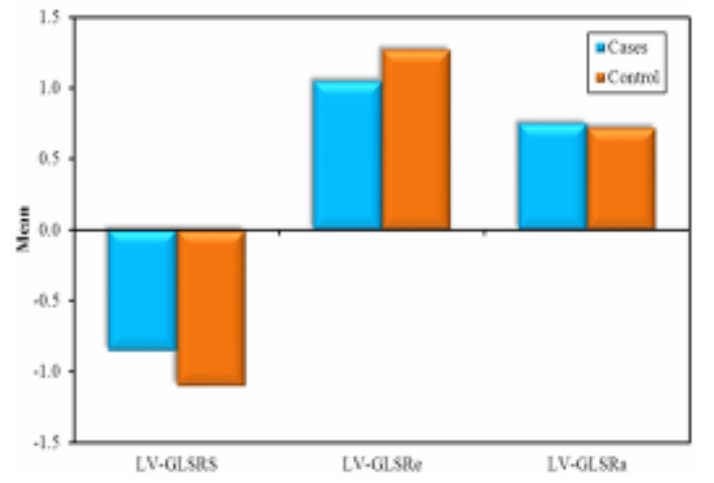

Figure 4: Comparison between the two studied groups according to LV-GLSRS, LV-GLSRe and LV-GLSRa

Correlations of LA-GLSRs, LA-GLSRe, LA-GLSRa, LV-GLSRs, LV-GLSRe and LVGLSRa with parameters of LA volume and function in NSTEMI patients were performed (Table 3).

Table 3: Correlation of global LA/LV strain rate parameters with LA volume and function parameters in patient with NSTEMI

\begin{tabular}{|l|l|c|c|c|c|c|}
\hline \multicolumn{2}{c|}{} & LAVmax(ml) & LAVmin(ml) & LAV res & LAEF(\%) & LVEF(\%) \\
\hline \multirow{2}{*}{ LA-GLSRs } & $\mathrm{r}$ & $-0.350^{*}$ & -0.339 & -0.259 & $-0.260^{*}$ & $-0.288^{*}$ \\
\cline { 2 - 7 } & $\mathrm{p}$ & $0.006^{*}$ & $0.008^{*}$ & $0.046^{*}$ & $0.044^{*}$ & $0.026^{*}$ \\
\hline \multirow{2}{*}{ LA-GLSRe } & $\mathrm{r}$ & 0.494 & 0.464 & 0.302 & -0.427 & -0.305 \\
\cline { 2 - 7 } & $\mathrm{p}$ & $<0.001^{*}$ & $<0.001^{*}$ & $0.019^{*}$ & $0.001^{*}$ & $0.018^{*}$ \\
\hline \multirow{2}{*}{ LA-GLSRa } & $\mathrm{r}$ & 0.387 & 0.346 & 0.259 & -0.102 & -0.063 \\
\cline { 2 - 7 } & $\mathrm{p}$ & $0.002^{*}$ & $0.007^{*}$ & $0.045^{*}$ & 0.439 & 0.633 \\
\hline \multirow{2}{*}{ LV-GLSRs } & $\mathrm{r}$ & 0.052 & 0.172 & -0.045 & -0.029 & -0.243 \\
\cline { 2 - 7 } & $\mathrm{p}$ & 0.691 & 0.188 & 0.735 & 0.826 & 0.062 \\
\hline \multirow{2}{*}{ LV-GLSRe } & $\mathrm{r}$ & -0.037 & -0.063 & 0.107 & 0.150 & 0.109 \\
\cline { 2 - 8 } & $\mathrm{p}$ & 0.778 & 0.631 & 0.416 & 0.253 & 0.409 \\
\hline \multirow{2}{*}{ LV-GLSRa } & $\mathrm{r}$ & -0.002 & 0.076 & -0.108 & 0.092 & 0.203 \\
\cline { 2 - 8 } & $\mathrm{p}$ & 0.988 & 0.564 & 0.413 & 0.484 & 0.120 \\
\hline
\end{tabular}

LA-GLSRs showed modest

correlations with parameters of LA volume and function, including LAVmax $(r=-0.350$, $\mathrm{p}=0.006)$, LAVmin $(\mathrm{r}=-0.339, \mathrm{p}=0.008)$, LAVres $(\mathrm{r}=-0.259, \mathrm{p}=0.046)$, LAEF $(\mathrm{r}=$ $0.260, p=0.044)$, and LVEF $(r=-0.288, p$ $=0.029)$. LA-GLSRe significantly correlated with LAVmax $(r=0.494, p<0.001)$, LAVmin $(\mathrm{r}=0.464, \mathrm{p}<0.001)$, LAVres $(\mathrm{r}=0.302, \mathrm{p}=$ 0.019), LAEF $(r=0.427, p=0.001)$, and LVEF $(\mathrm{r}=-0.305, \mathrm{p}<0.001)$. LA-GLSRa had significant correlations with the following echocardiographic variables: LAVmax $(\mathrm{r}=$ 0.387, $\mathrm{p}=0.002)$, LAVmin $(\mathrm{r}=0.346, \mathrm{p}<$
0.007), LAVres $(r=0.259, p=0.045)$, LAEF $(\mathrm{r}=-0.102, \mathrm{p}<0.439)$. and LVEF $(-0.063$, $\mathrm{p}=0.633)$ LV SR parameters had no significant correlation with the following LA echocardiographic variables: LAVmax, LAVmin, LAVres and LAEF. In addition, LVEF was significantly correlated with LAGLSRs $(r=-0.288, p=0.026)$ and LA-GLSRe $(\mathrm{r}=-0.305, \mathrm{p}=0.018)$ (Fig. 5), but not significantly correlated with LA-GLSRa $(\mathrm{r}=$ $0.063, \mathrm{p}=0.633)$. LA-GLSRe correlated significantly with LV-GLSRe $(\mathrm{r}=-0.226, \mathrm{p}=$ 0.04) (Fig. 6). 


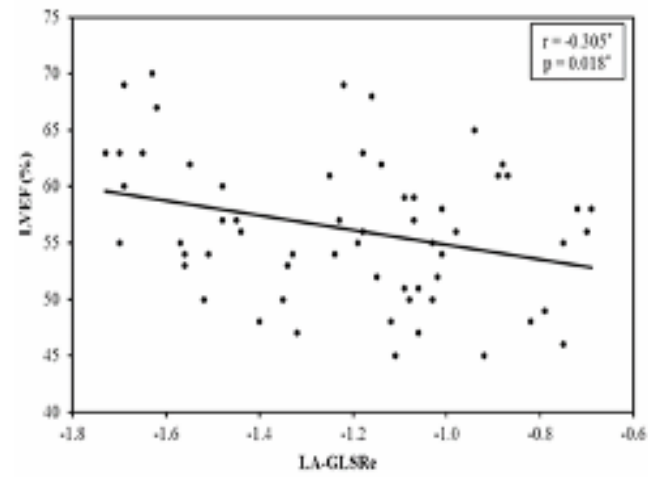

Figure 5: Correlation between left ventricular ejection fraction(LVEF) and peak early diastolic strain rate of the left atrium (LA-GLSRe) in patients with NSTEMI.

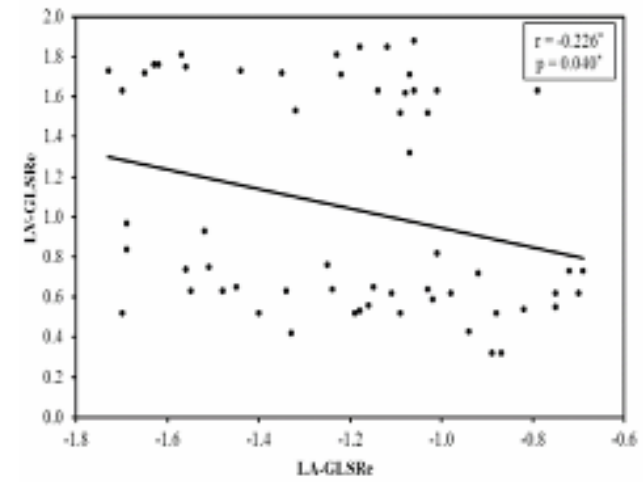

Figure 6: Correlation between peak early diastolic strain rate of the left ventricle (LVGLSRe) and peak early diastolic strain rate of the left atrium (LA-GLSRe) in patients with NSTEMI.

However, both LA-GLSRs and LA-GLSRa showed no such significant correlation with LVGLSRs (Fig. 7) and LV-GLSRa (Fig. 8), respectively.

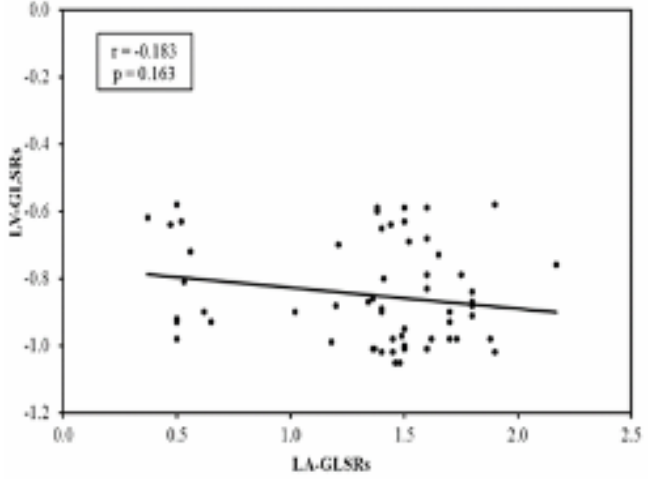

Figure 7: Correlation between peak early diastolic strain rate of the left ventricle (LVGLSRs) and peak early diastolic strain rate of the left atrium (LA-GLSRs) in patients with NSTEMI

\section{DISCUSSION}

\footnotetext{
After several decades of investigations, current consensus recommendations state that LA function plays an important role in optimizing overall cardiac function, and the changes in LA size and function are associated with cardiovascular disease and are risk factors for atrial fibrillation, stroke and death ${ }^{(\mathbf{1 7 )},(18)}$.

The left atrium serves as a blood reservoir during ventricular systole and a conduit for the passage of blood from the pulmonary veins into the left ventricle during early and middle ventricular diastole, as well as a booster pump increasing LV filling during late diastole ${ }^{(19)}$.
}



Figure 8: Correlation between peak early diastolic strain rate of the left ventricle (LV-GLSRa) and peak early diastolic strain rate of the left atrium (LA-GLSRa) in patients with NSTEMI.

In subjects with normal diastolic function, the relative contribution of the reservoir, conduit and pump function of the LA to the filling of the LV is approximately 40,35 and $25 \%$, respectively ${ }^{(20)}$.

Determined by conventional 2D echocardiography, LA function has been mainly evaluated using LA volumetric parameters and LA emptying fraction which may be used to evaluate the reservoir, conduit and booster pump components of LA function $^{(13),(21)}$.

Parameters that evaluate LA function may have prognostic potential. LA reservoir function may predict the first atrial fibrillation 
or flutter episode in elderly subjects, and LA systolic force may predict cardiovascular events in a population with a high prevalence of hypertension and diabetes ${ }^{(22),(23)}$. However, all these echocardiographic parameters and others that evaluate LA function are influenced by LV dynamics and geometry and/or rely on measurements that are subjected to error ${ }^{(\mathbf{2 4 )},(25)}$. Therefore, new methodologies that can evaluate LA function by analysis of LA myocardial deformation may be of potential clinical interest. Two strain imaging methods are based on different principles and can potentially give different results. Tissue Doppler imaging (TDI)-derived strain is limited to the measurement of movement parallel to the ultrasound beam. Non-Doppler 2D strain imaging derived from speckle tracking is a newer echocardiographic technique for obtaining SR measurements. The advantage of this method is that it tracks in two dimensions, along the direction of the wall, not along the ultrasound beam, and thus is angle independent, which is a great advantage of non-Doppler 2D strain imaging in comparison to TDI-derived strain data ${ }^{(2)}$. Previous studies show that 2D STE with its latest applications such as strain rate imaging may represent promising techniques to better evaluate LA function ${ }^{(8)}$. With the use of strain rate imaging, Inaba et al. found that SRs corresponded to reservoir function and SRe corresponded to conduit function, while SRa corresponded to booster pump function ${ }^{(\mathbf{2 6})}$. In patients with AMI, left ventricular stroke volume is relatively maintained despite the impairment of left ventricular function caused by myocardial ischaemia and necrosis. With increased stiffness or reduced compliance of the LV, LA pressure rises to maintain adequate LV filling, and the increased atrial wall tension leads to chamber dilatation and stretch of the atrial myocardium ${ }^{(27)}$. Therefore, the left atrium works harder and transports more blood to the left ventricle during left ventricular diastole. This function of the left atrium can be attributed to the Frank-Starling mechanism. LA pump function augmentation is therefore due to the increased left atrial volume before active atrial emptying, but not to the increased contractility of the left atrium ${ }^{(28)}$. In our study protocol, patients with NSTEMI showed increased LA volumes (LAVmax, LAVmin and LAVres). Moreover (LA total EF) was significantly impaired and compared with healthy controls. In accordance with the conventional echocardiographic parameters mentioned above, we found LA reservoir function assessed by SR imaging (LA-GLSRs) and LA conduit function assessed by SR imaging (LA-GLSRe) were significantly reduced in patients with NSTEMI (Table 2), but LA booster function assessed by SR imaging (LA-GLSRa) showed no significant difference. This may be explained by when the LA is well stretched longitudinally, and consequently a high LA positive peak is present, the LV then relaxes rapidly, generating a high $\mathrm{E}$ wave, as blood rushes into the LV, generating a high passive LA emptying fraction. Therefore, LA-GLSRs and/or LA-GLSRe have significant correlations with LV diastolic function, which are impaired in patients with NSTEMI. In our study protocol, a good correlation was found between LA global strain rate and LA functional parameters (Table 3). The present study extends previous results and describes changes in LA function after AMI, combining LA volumes, LA emptying fraction, and LA strain in patients with NSTEMI. The results show that speckle tracking-derived strain rate is a promising technique to assess LA function as well as LA volumes and LA emptying fraction. Global strain is a relatively new parameter for assessment of LV function ${ }^{(29)}$ and tends to predict the infarct mass better than established indices of global function such as LVEF and WMSI. LVEF can be regarded as the sum of all LV systolic deformation.

In Wakami et al.'s study, peak LA strain rate during LV systole, which corresponds to our measured LA-GLSRs, correlated inversely with LV end-diastolic pressure and LV end-systolic volume and positively with $\mathrm{LVEF}^{(30)}$. In a recent study by Vartdal et al. global strain measured by TDI immediately after PCI was found to be superior to LVEF for predicting final infarct mass in patients with acute $\mathrm{MI}^{(\mathbf{3 0})}$. Comparing with tagged magnetic resonance imaging (the current 'gold standard' for deformation analysis), STE measurements correlated well with data obtained by magnetic resonance imaging, both in normal myocardial segments and infarcted areas $(r=0.87, p<0.001)^{(30)}$. The findings of our present study are in accordance with previous studies. There was 
significant correlation between LVEF and global LA-GLSRs $(r=-0.334, p<0.05)$ or LA-GLSRe $(r=-0.477, p<0.001)$. In particular, LA-GLSRe was strongly correlated with LV-GLSRe $(r=-0.644, p=0.001)$, while LA-GLSRs and LA-GLSRa were not significantly correlated with LV strain rate parameters (LV-GLSRs and LV-GLSRa). These findings support the idea that LAGLSRe can serve as an important new marker of LA and LV function in the acute MI. Therefore, speckle tracking echocardiography was found to be a feasible and reproducible method to assess LA longitudinal strain in healthy subjects and patients with NSTEMI. The reproducibility of measurements was good, with lower variability of intra- and interobserver. In particular, we found LA-GLSRe was significantly correlated with both LA 2D Doppler echocardiographic parameters and LV contractile function, and could be an optimal parameter of 2D STE in assessing the degree of impairment of heart function in patients with NSTEMI.

These data suggest that speckle tracking echocardiography may be considered a promising tool to explore LA myocardial deformation dynamics

\section{Study limitations}

A number of obvious limitations of our study should be noted. First, the 2D STE analysis software that was originally designed for the left ventricle was applied to the left atrium in our study. Second, echocardiography in this study was not performed in the emergency room but on arrival at the coronary care unit or one to two days later. Third, the relatively small number of patients eligible for analysis in the present study may render it difficult to generalise the results and apply them to other patient populations. Further larger, prospective studies are required to determine the cost effectiveness of this new technique to evaluate LA function in NSTEMI patients. Lastly, this was a cross-sectional study, and therefore no clinical outcomes were examined.

\section{CONCLUSIONS}

Our study demonstrated that twodimensional speckle tracking echocardiography represented a non-invasive, relatively simple and reproducible technique to assess left atrial myocardial function in patients with NSTEMI. The reservoir and conduit function of the left atrium were impaired in these patients, compared with controls, LA-GLSRe was significantly correlated with both LA 2D Doppler echocardiographic parameters and LV contractile function and could be suggested as a better indicator to evaluate LA function as a preferred parameter of STE.

\section{REFERENCES}

1. Thygesen K, Alpert JS, Jaffe AS, Simoons ML, Chaitman BR, White HD et al. (2012): Third universal definition of myocardial infarction. European Heart Journal, 33(20): 255167.

2. Perk G, Kronzon I (2009): Non-Doppler Two-Dimensional Strain Imaging for Evaluation of Coronary Artery Disease. Echocardiography, 26(3):299-306.

3. Blume GG, Mcleod CJ, Barnes ME, Seward JB, Pellikka PA, Bastiansen PM et al. (2011): Left atrial function: physiology, assessment, and clinical implications. European Journal of Echocardiography, 12(6):421-30.

4. Abhayaratna WP, Seward JB, Appleton CP, Douglas PS, Oh JK, Tajik AJ et al. (2006): Left Atrial SizePhysiologic Determinants and Clinical Applications. Journal of the American College of Cardiology, 47(12):235763.

5. Stefanadis C, Dernellis $\mathbf{J}$, Toutouzas $\mathbf{P}$ (2001): A clinical appraisal of left atrial function. European heart journal, 22(1):22-36.

6. Dandel M, Knosalla C, Lehmkuhl H, Hetzer R (2007): Non-Doppler Twodimensional Strain Imaging-Clinical Applications. Journal of the American Society of Echocardiography, 20(8):1019. 
7. Kim DG, Lee KJ, Lee S, Jeong SY, Lee YS, Choi YJ et al. (2009): Feasibility of Two-Dimensional Global Longitudinal Strain and Strain Rate Imaging for the Assessment of Left Atrial Function: A Study in Subjects with a Low Probability of Cardiovascular Disease and Normal Exercise Capacity. Echocardiography, 26(10):1179-87.

8. Saraiva RM, Demirkol S, Buakhamsri A, Greenberg N, Popović ZB, Thomas JD et al. (2010): Left atrial strain measured by two-dimensional speckle tracking represents a new tool to evaluate left atrial function. Journal of the American Society of Echocardiography, 23(2):172-80.

9. Leung DY, Boyd A, Ng AA, Chi C, Thomas L (2008): Echocardiographic evaluation of left atrial size and function: current understanding, pathophysiologic correlates, and prognostic implications. American Heart Journal, 156(6):1056-64.

10. Reisner SA LP, Agmon Y et al. (2004): Global longitudinal strain: anovel index of left ventricular systolic function. J Am Soc Echocardiogr., 17: 630-3.

11. Sirbu C, Herbots L, D'hooge J, Claus P, Marciniak A, Langeland $\mathrm{T}$ et al. (2006): Feasibility of strain and strain rate imaging for the assessment of regional left atrial deformation: a study in normal subjects. European Journal of Echocardiography, 7(3):199-208.

12. Eshoo S, Boyd AC, Ross DL, Marwick TH, Thomas L (2009): Strain rate evaluation of phasic atrial function in hypertension. Heart, 95(14):1184-91.
13. Lang RM, Badano LP, Mor-Avi V, Afilalo J, Armstrong A, Ernande $L$ et al. (2015): Recommendations for cardiac chamber quantification by echocardiography in adults: an update from the American Society of Echocardiography and the European Association of Cardiovascular Imaging. Journal of the American Society of Echocardiography, 28(1):139. e14.

14. Dogan C, Ozdemir N, Hatipoglu S, Bakal RB, Omaygenc MO, Dindar B et al. (2013): Relation of left atrial peak systolic strain with left ventricular diastolic dysfunction and brain natriuretic peptide level in patients presenting with ST-elevation myocardial infarction. Cardiovascular ultrasound, 11(1):24.

15. Serri K RP, Lafitte $M$ et al. (2006): Application in hypertrophic,: c. Global and regional myocardial function quantification by two-dimensional strain; J Am Coll Cardiol., 47:117581.

16. Cerqueira MD WN, Dilsizian $\mathrm{V}$ et al. (2002): Standardized myocardial segmentation and nomenclature for tomographic imaging of the heart Imaging. Circulation, 105:32-35.

17. Sanfilippo AJ, Abascal VM, Sheehan M, Oertel LB, Harrigan P, Hughes RA et al. (1990): Atrial enlargement as a consequence of atrial fibrillation. A prospective echocardiographic study. Circulation, 82(3):792-7.

18. Vaziri SM, Larson MG, Benjamin EJ, Levy D (1994): Echocardiographic predictors of nonrheumatic atrial fibrillation. The Framingham Heart Study. Circulation, 89(2):724-30.

19. Abhayaratna WP, Seward JB, Appleton CP, Douglas PS, Oh JK, Tajik AJ et al. (2006): Left atrial size: physiologic determinants and clinical applications. Journal of the American College of Cardiology, 47(12):2357-63. 
20. Prioli A, Marino P, Lanzoni L, Zardini $P$ (1998): Increasing degrees of left ventricular filling impairment modulate left atrial function in humans. American Journal of Cardiology, 82(6):756-61.

21. Gutman J, Wang YS, Wahr D, Schiller NB (1983): Normal left atrial function determined by 2-dimensional echocardiography. The American Journal of Cardiology, 51(2):336-40.

22. Abhayaratna WP, Fatema $K$, Barnes ME, Seward JB, Gersh BJ, Bailey KR et al. (2008): Left atrial reservoir function as a potent marker for first atrial fibrillation or flutter in persons $\geq$ 65 years of age. American Journal of Cardiology, 101(11):1626-9.

23. Chinali M, Simone Gd, Roman MJ, Bella JN, Liu JE, Lee ET et al. (2005): Left atrial systolic force and cardiovascular outcome: The Strong Heart Study. American journal of hypertension, 18(12):1570-6.

24. Suh IW, Song JM, Lee EY, Kang SH, Kim MJ, Kim JJ et al. (2008): Left atrial volume measured by real-time 3dimensional echocardiography predicts clinical outcomes in patients with severe left ventricular dysfunction and in sinus rhythm. Journal of the American Society of Echocardiography, 21(5):439-45.

25. Maddukuri PV, Vieira ML, DeCastro S, Maron MS, Kuvin JT, Patel AR et al. (2006): What is the best approach for the assessment of left atrial size? Comparison of various unidimensional and two-dimensional parameters with three-dimensional

echocardiographically determined left atrial volume. Journal of the American Society of Echocardiography, 19(8):1026-32.
26. Inaba $\mathbf{Y}$, Yuda $\mathrm{S}$, Kobayashi $\mathbf{N}$, Hashimoto A, Uno K, Nakata $T$ et al. (2005): Strain rate imaging for noninvasive functional quantification of the left atrium: comparative studies in controls and patients with atrial fibrillation. Journal of the American Society of Echocardiography, 18(7):729-36.

27. Vianna-Pinton R, Moreno CA, Baxter CM, Lee KS, Tsang TS, Appleton CP (2009): Two-dimensional speckletracking echocardiography of the left atrium: feasibility and regional contraction and relaxation differences in normal subjects. Journal of the American Society of Echocardiography, 22(3):299-305.

28. Matsuda $Y$, Toma $Y$, Ogawa $H$, Matsuzaki M, Katayama K, Fujii T et al. (1983): Importance of left atrial function in patients with myocardial infarction. Circulation, 67(3):566-71.

29. Peteiro J, Garcia-Lara J, Garrido I, Monserrat L, Aldama G, CastroBeiras A (2005): A new simple method to assess global left ventricular systolic function based on the sum of regional myocardial velocities. American Journal of Cardiology, 95(4):550-2.

30. Wakami K, Ohte N, Asada K, Fukuta H, Goto T, Mukai S et al. (2009): Correlation between left ventricular end-diastolic pressure and peak left atrial wall strain during left ventricular systole. Journal of the American Society of Echocardiography, 22(7):847-51. 